\title{
Estrategias metodológicas para mejorar el trabajo cooperativo en estudiantes de educación secundaria, Cajamarca, 2020
}

\author{
Methodological strategies to improve cooperative work in high school students, \\ Cajamarca, 2020
}

Juan Romelio, Mendoza Sánchez «x 1,a

https://orcid.org/0000-0002-1690-8537

Blanca Flor, Mendoza Sánchez 1,b

https://orcid.org/0000-0002-4665-7097

\section{Citar como}

Mendoza Sánchez, J. y Mendoza Sánchez, B. (2021). Estrategias metodológicas para mejorar el trabajo cooperativo en estudiantes de educación secundaria, Cajamarca, 2020. Desafíos, 12(1); 44-9. https://doi.org/10.37711/desafios.2021.12.1.254

\section{RESUMEN}

Objetivo. Identificar las estrategias de trabajo cooperativo en los alumnos de formación secundaria de la ciudad de Cajamarca. Métodos. El estudio tuvo un enfoque cualitativo, con diseño no experimental, transeccional y fue de tipo descriptivo. Se utilizó como instrumentos el cuestionario, así como también la lista de cotejos; en cuanto al instrumento, este fue validado mediante el juicio de expertos con un cociente de valoración de $90 \%$. Se estableció la confiabilidad mediante el coeficiente Kuder-Richardson, el cual fue igual a 0,83 y 0,81; por lo que se puede mencionar que el instrumento es de muy alta confiabilidad. Resultados. La estrategia metodológica de trabajo cooperativo mediante sus cuatro fases: programación, operación, reflexión y reflexión, y las metodologías Puzzle o la averiguación por grupos, permiten viabilizar condiciones favorables al trabajo cooperativo entre los alumnos. Conclusiones. Se comprueba la hipótesis de que una adecuada estrategia metodológica crea un apropiado aprendizaje, beneficiando de este modo las estrategias metodológicas de los alumnos.

Palabras clave: estrategias metodológicas; trabajo cooperativo.

\section{ABSTRACT}

Objective. To identify cooperative work strategies in high school students in the city of Cajamarca. Methods. The study had a qualitative approach, with a non-experimental, transectional and descriptive design. The instruments used were the questionnaire and the checklist; the instrument was validated by means of expert judgment with a valuation quotient of $90 \%$. Reliability was established by means of the Kuder-Richardson coefficient, which was equal to 0.83 and 0.81 ; therefore, it can be said that the instrument has a very high reliability. Results. The methodological strategy of cooperative work through its four phases: programming, operation, reflection and reflection, the Puzzle methodologies and the inquiry by groups, make it possible to create favorable conditions for cooperative work among students. Conclusions. The hypothesis that an adequate methodological strategy creates an appropriate learning process, thus benefiting the methodological strategy of the students, is proved.

Keywords: methodological strategies; cooperative work.

\footnotetext{
Filiación y grado académico

1 Universidad Nacional de Cajamarca, Perú.

a Maestro en ciencias, mención Investigación y Docencia.

b Master en Dirección y consultoría turística.
} 


\section{INTRODUCCIÓN}

El presente artículo se asienta en uno de los problemas más característicos y frecuentes en los establecimientos educativos, especialmente en Perú, donde se evidencia según las deficiencias en el estilo de trabajo en los alumnos de secundaria y, con ello, sus variadas consecuencias; no sólo en el rendimiento del estudiante mismo, sino también en el perfeccionamiento de su idiosincrasia y autoestima. Problema ya acarreado, según Skinner, desde la década de los setenta. Por lo tanto, hemos considerado los siguientes antecedentes referidos al estudio.

En su tesis "Las estrategias metodológicas utilizadas por el profesor de matemática en la enseñanza media y su relación con el desarrollo de habilidades intelectuales de orden superior en sus alumnos", Matamala (2008) desarrolló un diseño no experimental transeccional de tipo descriptivo, concluyendo que ni las estrategias metodológicas, ni las escrituras de estimación de los pedagogos originan el proceso insondable de la investigación en el estudiante.

En tanto Huertas (2005), en su tesis "Aprendizaje estratégico en el rendimiento académico de los alumnos de la Facultad de Educación", da centralidad al aspecto metódico de la instrucción estratégica en el rendimiento académico. Su estudio concluye que las estrategias de aprendizaje asentado la actividad en el rendimiento académico y la diligencia de disímiles esquemas cognoscitivos como mapas semánticos, conceptuales, mentales, redes semánticas, etc., conformándose como técnicas muy eficaces en el procesamiento de la información, ordenamiento, asimilación y comprensión del conocimiento.

Considerando todas estas ideas patentizamos la calidad que posee el trabajo cooperativo en los centros educativos. Tal como lo señala Bandura (1977), el trabajo cooperativo es muy limitado, puesto que sólo nos consignamos a conceptos restrictivos y elitistas como la acumulación de individuos establecidos para una prestación o indagación concluyente. Así tambien Coll (1984) señalaba que el trabajo cooperativo lo logramos concebir a modo de congregación de individuos que emplazan sus esfuerzos para conseguir resultados placenteros en el mando de un contenido o compromiso común; en tanto Ander (1996) proyecta el subsiguiente concepto:

Se trata de un conjunto de personas que tienen un alto nivel de capacidad operativa de cara al logro de determinados objetivos y a la realización de actividades orientadas a la consecución de los mismos. El trabajo individual y colectivo se realiza con un espíritu de complementación, mediante una adecuada coordinación y articulación de tareas, y en un clima de respeto y confianza mutua altamente satisfactorio.

En este escenario Enríquez (1999), señala que en la cultura de la cooperación es fundamental la premisa holística de los maestros para posesionarse de diferentes roles formativos de los estudiantes. Por lo tanto, la investigación facto-perceptible evidenció como problema que los estudiantes de educación secundaria de la localidad de Cajamarca presentan una operante experiencia de trabajo cooperativo.

El objeto de la investigación es vislumbrar el juicio de enseñanza-aprendizaje en correspondencia al trabajo cooperativo en educación secundaria. El objetivo planteado en el presente estudio, por lo tanto, es: diseñar estrategias metodológicas participativas sustentadas en la teoría Z de W. Ouchi y la teoría de las relaciones interpersonales, las cuales, de acuerdo a Fabra (1992), para optimizar el trabajo cooperativo en los alumnos es necesario diseñar nuevas estrategias de trabajo cooperativo; este enunciado fue tratado también, con posteridad, por Johnson y Johnson en el año de 1999.

La hipótesis a confirmar es: si se diseñan estrategias metodológicas participativas respaldadas en la teoría Z de William Ouchi y en la teoría de las relaciones interpersonales, entonces es posible perfeccionar el trabajo cooperativo en los alumnos del primer grado de educación secundaria de la localidad de Cajamarca.

La contribución primordial de la investigación estriba en la elaboración de estrategias metodológicas participativas de trabajo cooperativo para destacar el incorrecto estilo de trabajo entre los alumnos de la Institución educativa.

Los Objetivos específicos consisten en: efectuar un análisis situacional para tratar de ver las peculiaridades del trabajo áulico desarrollado por los alumnos; exponer la relación entre las variables y el marco teórico de la indagación, y plantear en ultima instancia estrategias metodológicas interactivas para optimizar el trabajo cooperativo en los alumnos del primer grado de educación secundaria de la ciudad de Cajamarca.

\section{MÉTODOS}

\section{Tipo de estudio}

Considerando el aspecto metodológico, el presente estudio parte de la perspectiva del 
paradigma socio-cognitivo enmarcado en un tipo de investigacion descriptiva con un diseño no experimental, Transeccional.

\section{Población muestral}

Fueron 23 alumnos del nivel secundario, correspondiente al año 2020. Se incluyó a alumnos de primer grado de un establecimiento educativo privado por tener acceso a la aplicación del instrumento, de los cuales 11 fueron mujeres y 12 varones, asimismo, se excluyeron las demás instituciones y grados respectivos por no formar parte del estudio.

\section{Instrumentos de medición}

Los instrumentos de recolección de datos fueron seleccionados de acuerdo a las variables, tomando en consideración la técnica de la encuesta, análisis de documentaciones y como instrumento para la recaudación de información se utilizó el cuestionario y la guía de análisis documental, el cuestionario fue elaborado por los responsables de la investigación el cual consigno un acumulado de preguntas concernientes a los indicadores de cada una de las dimensiones de las variables señaladas, con sus respectivas alternativas, las que fueron valoradas de acuerdo a los juicios estadísticos elementales. El cuestionario fue validado a través del juicio de expertos, con un promedio de valoración de 90 \%. Se determinó la confiabilidad a través del coeficiente Kuder-Richardson resultando igual a 0,83 y 0,81 , por lo que el instrumento resulta de muy alta confiabilidad.

\section{Procedentes de recolección de datos}

En cuanto al procedimiento de recolección de datos, se consideraron las técnicas de la estadística descriptiva, así también tablas de frecuencia, en representación de frecuencias estrictas y porcentuales, además se utilizó la tabla de contingencia para concebir la distribución de las identificaciones según los niveles.

\section{Análisis de datos}

El análisis de datos fue realizado de acuerdo a las respuestas obtenidas complementando con la observación de los investigadores para comprobar que una apropiada estrategia metodológica genera un adecuado aprendizaje.

\section{Aspectos éticos}

Referente a los aspectos éticos de la investigación, se está citando a todas las fuentes que han sido consultadas y consideradas; también contamos con la autorización de la institución en estudio para recolectar la información necesaria. Dicha información fué usada exclusivamente con fines académicos, basándonos en el método científico y sin dejar de lado valores que un investigador debe observar. Finalmente, todos los resultados se presentan sin alterar datos reales.

\section{RESULTADOS}

A continuación, se muestran los resultados de la investigación tomando en consideración la población de estudio en relación a los indicadores de los instrumentos aplicados a los estudiantes.

En la siguiente tabla se consigue representar que 8 estudiantes varones que corresponde a un $35 \%$, tienen predilecciones para trabajar con alguien en específico dentro del aula de clase y únicamente 3 estudiantes, lo equivalente al $13 \%$, no poseen predilección por alguno en especial para trabajar dentro del aula. En cuanto a las damas, en un $52 \%$ tiene predilección por alguien en especial para trabajar dentro del aula y, simplemente, una de ellas indicó que, a veces, tiene predilecciones por alguien para trabajar en su aula. Por tanto, a propósito de esta tabla podemos expresar que los estudiantes hombres de educación secundaria presentan, en su mayoría, preferencia por alguien en especial para trabajar dentro del aula y las damas de la misma manera, en un 52 \% tienen esta preferencia; lo cual es excluyente para el trabajo apropiado en el aula (ver tabla 1).

\section{Tabla 1}

Preferencias por trabajar con alguien en especial adentro de su aula

\begin{tabular}{|c|c|c|c|c|c|c|}
\hline \multirow{2}{*}{$\begin{array}{l}\text { Tienes preferencias por trabajar con alguien } \\
\text { en especial dentro su aula }\end{array}$} & \multicolumn{2}{|c|}{ Varones } & \multicolumn{2}{|c|}{ Damas } & \multicolumn{2}{|c|}{ Total } \\
\hline & $\mathrm{fi}$ & $\%$ & $\mathrm{fi}^{\circ}$ & $\%$ & fi & $\%$ \\
\hline Sí & 08 & 35 & 11 & 52 & 19 & 100 \\
\hline No & 03 & 13 & 00 & 00 & 03 & 00 \\
\hline A veces & 00 & 00 & 01 & 04 & 01 & 04 \\
\hline Total & 11 & 48 & 12 & 52 & 23 & 100 \\
\hline
\end{tabular}


Tabla 2

¿En su opinión quien es para usted el compañero, de su agrado para trabajar dentro de su aula?

\begin{tabular}{|c|c|c|c|c|c|c|}
\hline \multirow{2}{*}{$\begin{array}{l}\text { En su opinión quien es para usted el compañero de } \\
\text { su agrado para trabajar dentro de su aula }\end{array}$} & \multicolumn{2}{|c|}{ Varones } & \multicolumn{2}{|c|}{ Damas } & \multicolumn{2}{|c|}{ Total } \\
\hline & fi & $\%$ & fi & $\%$ & $\mathrm{fi}^{\circ}$ & $\%$ \\
\hline Mi mejor amigo del aula & 05 & 22 & 10 & 43 & 15 & 65 \\
\hline El mejor alumno del aula & 05 & 22 & 02 & 9 & 7 & 31 \\
\hline Cualquier compañero de aula & 01 & 04 & 00 & 00 & 1 & 04 \\
\hline Total & 11 & 48 & 12 & 52 & 23 & 100 \\
\hline
\end{tabular}

En la siguiente tabla, se logra visualizar que para 5 alumnos hombres, el equivalente al $22 \%$ el acompañante de su agrado para trabajar en el aula es su mejor compañero, en semejante porcentaje opinaron que el mejor compañero para trabajar en el aula es el mejor estudiante del aula y 1 el equivalente a $4 \%$, señalo que cualquier compañero es de su agrado para trabajar dentro de su aula. Respecto a las damas, 10 de ellas, lo que viene a ser el equivalente a un $43 \%$, aludieron que el compañero de su preferencia para trabajar en su aula es su mejor amigo; 2 de ellas, o el equivalente al $9 \%$, indicaron que la persona de su agrado para trabajar en el aula es el mejor estudiante del aula (ver tabla 2).

En la siguiente tabla, podemos observar que 04 estudiantes varones, equivalentes al $17 \%$, se interrelacionaron apropiadamente cuando existe trabajos en equipo, otros 5 , que vendrían a ser $22 \%$, se interrelacionan cuando hay acciones de congregación; y únicamente el 9 \% se congregan por otras situaciones. En cuanto a las damas el $13 \%$ se interrelacionan convenientemente cuando hay trabajos en equipo, el $35 \%$ se interrelaciona cuando hay actividades de fraternidad y el $4 \%$ se congrega por otras situaciones. Por lo tanto, en relación a esta tabla podemos expresar que los estudiantes hombres se interrelacionan en su mayoría por interés; es decir exclusivamente porque existen trabajos en equipo o actividades de fraternidad. Asimismo, las damas se interrelacionan por las mismas razones, lo cual hace conjeturar que no existe un apropiado trabajo en equipo en esta institución educativa y particularmente, en el aula del primer grado del nivel secundaria (ver tabla 3).

En la siguiente tabla, se demuestra que 3 estudiantes varones, el equivalente al $13 \%$, intervinieron para solucionar algún inconveniente entre compañeros, pero la mayoría de estos estudiantes, el $35 \%$, no intervino nunca para ayudar a solucionar cierto problema entre compañeros. En cuanto a las damas, el 17 \% sí intervino como intermediario para solucionar algún problema entre compañeros pero al igual que los hombres la mayoría representado por el 52 \% nunca intervino como mediador para ayudar a solucionar algún problema entre. Por lo tanto, podemos inferir de este cuadro que los alumnos en su mayoría son indiferentes a los problemas de sus compañeros; lo cual hace conjeturar que existe penuria del valor solidaridad (ver tabla 4).

\section{Tabla 3}

¿En qué circunstancias cree usted haber cooperado adecuadamente con alguno(a) de sus compañeros de aula?

\begin{tabular}{|c|c|c|c|c|c|c|}
\hline \multirow{2}{*}{$\begin{array}{l}\text { En qué circunstancias cree usted haber } \\
\text { cooperado adecuadamente con alguno(a) } \\
\text { de sus compañeros de aula }\end{array}$} & \multicolumn{2}{|c|}{ Varones } & \multicolumn{2}{|c|}{ Damas } & \multicolumn{2}{|c|}{ Total } \\
\hline & fi & $\%$ & fi & $\%$ & $\mathrm{fi}^{\circ}$ & $\%$ \\
\hline Cuando hay trabajos grupales por realizar & 04 & 17 & 03 & 13 & 07 & 30 \\
\hline $\begin{array}{l}\text { Cuando han tenido problemas dentro o } \\
\text { fuera del aula }\end{array}$ & 00 & 00 & 00 & 00 & 00 & 00 \\
\hline $\begin{array}{l}\text { Cuando hay actividades de confraternidad } \\
\text { dentro de la IE }\end{array}$ & 05 & 22 & 08 & 35 & 13 & 57 \\
\hline Otros & 02 & 09 & 01 & 04 & 03 & 13 \\
\hline Total & 11 & 48 & 12 & 52 & 23 & 100 \\
\hline
\end{tabular}


Tabla 4

¿Dígame una circunstancia en el que usted cooperó para ayudar alguno (a) de sus compañeros (as) a resolver algún problema?

\begin{tabular}{|c|c|c|c|c|c|c|}
\hline \multirow{2}{*}{$\begin{array}{l}\text { Dígame una circunstancia en el que usted } \\
\text { coopero para ayudar alguno (a) de sus } \\
\text { compañeros (as) a resolver }\end{array}$} & \multicolumn{2}{|c|}{ Varones } & \multicolumn{2}{|c|}{ Damas } & \multicolumn{2}{|c|}{ Total } \\
\hline & fi & $\%$ & fi & $\%$ & fi & $\%$ \\
\hline Cuando hubo problemas entre compañeros & 03 & 13 & 04 & 17 & 7 & 30 \\
\hline Cuando hubo problemas con algún docente & 00 & 00 & 00 & 00 & 00 & 00 \\
\hline Nunca & 08 & 35 & 08 & 35 & 16 & 70 \\
\hline Total & 11 & 48 & 12 & 52 & 23 & 100 \\
\hline
\end{tabular}

Nota: Encuesta aplicada a estudiantes de educación secundaria.

Como podemos observar en la siguiente tabla, 3 estudiantes varones, el equivalente al $13 \%$ demostraron sus relaciones interpersonales apoyando en tareas que vislumbran sus compañeros: así también, existen 3 de estudiantes que demuestran las relaciones interpersonales convenientes, siendo copartícipes y apoyando en la solución de problemas y otros 5 estudiantes manifestaron lo mismo, impidiendo conflictos con sus compañeros. En cuanto a las damas, el $17 \%$ manifestó sus buenas relaciones interpersonales apoyando a sus compañeros en tareas que no entendieron; el $22 \%$ manifestaron sus buenas relaciones interpersonales, siendo responsables y apoyando a sus demás compañeros cuando tienen problemas y un 13 \% señalan las adecuadas relaciones interpersonales impidiendo conflictos. De modo que los estudiantes tratan de tener buenas relaciones, pero por diferentes impulsos, desde el apoyo en tareas hasta prescindiendo conflictos, pero quienes más ejercen obviamente son las mujeres (ver tabla 5).

\section{DISCUSIÓN}

En el presente artículo se ha efectuado, de modo preciso, los objetivos trazados, instaurando la correspondencia entre variables. Así también se evidenció que, en la actualidad, la diligencia de instruir y aprender una ocupación tiene que estar ligada al progreso del pensamiento.
Son las presunciones constructivistas las que sostienen que, la reconstrucción de los aprendizajes no es fruto de la mera transferencia y aceptación de averiguación, sino que, más bien, se da a través de la interacción activa entre el educador y los estudiantes. Sin embargo, por lo general en los establecimientos educativos aún prevalecen sistemas comprensibles tradicionales donde se ignora el uso de estrategias y técnicas que ayuden al perfeccionamiento de los aprendizajes de los estudiantes.

Por tal razón, concordamos con Matamala (2008), quien concluye en la tesis: "Las estrategias metodológicas utilizadas por el profesor de matemática en la enseñanza media y su relación con el desarrollo de habilidades intelectuales de orden superior en sus alumnos". Quien, concluye que, ni las estrategias metodológicas, ni las formas de evaluación de los profesores actuales suscitan el procesamiento insondable de la averiguación en el estudiante.

Asimismo, concordamos con Huertas (2005), quien en su tesis: "Aprendizaje estratégico en el rendimiento académico de los alumnos de la Facultad de Educación", quien señala que las estrategias de aprendizaje deben estar basadas en el rendimiento académico y la aplicación de disímiles bosquejos cognitivos como el mapa semántico, conceptual, mental, redes semánticas, etc. Estos estudios, al igual que el nuestro, están reforzados por las teorías de clásicos como Skinner (1977), quien manifiesta

Tabla 5

¿Cómo usted mostraría a sus compañeros de aula la importancia de las relaciones interpersonales?

\begin{tabular}{|c|c|c|c|c|c|c|}
\hline \multirow{2}{*}{$\begin{array}{l}\text { Cómo usted mostraría a sus compañeros de aula la } \\
\text { importancia de las relaciones interpersonales }\end{array}$} & \multicolumn{2}{|c|}{ Varones } & \multicolumn{2}{|c|}{ Damas } & \multicolumn{2}{|c|}{ Total } \\
\hline & fi & $\%$ & fi & $\%$ & fi & $\%$ \\
\hline Apoyándolo en sus tareas que no comprendieron & 03 & 13 & 04 & 17 & 7 & 30 \\
\hline $\begin{array}{l}\text { Siendo solidarios y apoyándolos cuando tienen } \\
\text { problemas }\end{array}$ & 03 & 13 & 05 & 22 & 08 & 35 \\
\hline $\begin{array}{l}\text { Evitando conflictos con ellos y entre los demás } \\
\text { compañeros de aula }\end{array}$ & 05 & 22 & 03 & 13 & 08 & 35 \\
\hline TOTAL & 11 & 48 & 12 & 52 & 23 & 100 \\
\hline
\end{tabular}

Nota: Encuesta aplicada a estudiantes de educación secundaria. 
que las deficiencias en el estilo de trabajo en los estudiantes no sólo se presenta en el rendimiento del alumno mismo, sino también en el desarrollo de su personalidad y autoestima; por ello, como menciona Lewin (1973), se debe promover en el estudiante dinámicas de personalidad, lo cual es erróneo tal y como ocurre en nuestro contexto educativo, pues a pesar que los docentes han sido entrenados en el uso de nuevas estrategias de enseñanza y aprendizajes, éstos no emplean el aprendizaje cooperativo. De ningún modo existen indagaciones sobre esta materia. De ahí que la importancia de este estudio radica en contribuir con el diseño de una estrategia metodológica participativa, propensa a optimizar el estilo de trabajo en equipo en los estudiantes de educación del nivel secundario de la localidad de Cajamarca.

Para ello se despliega la fase diagnóstica, para tratar de ver y comprender el contexto áulicoinstitucional que se intenta optimar y poder determinar la eficacia de las operaciones y estrategias a utilizar. Es necesario también señalar que, por el momento, son bastante escasas las investigaciones que aborden las estrategias metodológicas para mejorar el trabajo cooperativo en alumnos del nivel secundario en nuestra localidad y algunas de éstas no se encuentran a disposición inmediata o libre. Además, existe poca disponibilidad de información sobre datos específicos respecto al alcance, frecuencia y el impacto del trabajo cooperativo.

En cuanto a las implicancias del estudio podemos mencionar que es relevante, porque dicha investigación puede ser sometida a validez y confiabilidad, constituyéndose en una propuesta que puede aplicarse a en contextos similares del sector educación y, a su vez, servir de antecedente para otros estudios con variables similares.

Así también, mencionaremos que para el presente estudio se presentaron limitaciones exclusivamente en relación a estudios retrospectivos, puesto que este tema no ha sido tratado durante los últimos tiempos. Por ello es que esta investigacion presenta estudios no extrapolables.

Finalmente, y luego de obtener nuestros resultados, señalamos que las estrategias metodológicas para optimizar el trabajo cooperativo en estudiantes del nivel Secundario en Cajamarca, 2020, están vinculadas a nuestros antecedentes y teorías estudiadas; por lo que recomendamos a los docentes tener predisposición práctica para desarrollar los estilos de trabajo en equipo en las aulas, dado que en ello radica la importancia del trabajo y la contingencia de que su aplicación y profundización teórica se haga viable a fin de enriquecer el mismo.

\section{Agradecimientos}

Un sincero y especial agradecimiento de mi persona a la iluminación y fuerza de Rafael Mendoza Sánchez; gracias por ser la persona con quien más me he enfadado, he lidiado, etc. Pero, del mismo modo, con quien más me he entendido. Gracias por todo, querido y nunca olvidado hermano.

\section{REFERENCIAS}

Ander, E. (1996). Buscando la sinergia en Trabajo Social: Técnicas de Reuniones de Trabajo. Colatina

Bandura, A. (1977). Self-Efficacy: Toward a unifying theory of behavioral change. Psychological Review, 84(2), 191-215.

Coll, C. (1984). Estructura grupal, interacción entre alumnos y aprendizaje escolar. Journal for the Study of Education and Development, Infancia y Aprendizaje, 27-28, 119-138.

Enríquez, L. (1999). El aprendizaje cooperativo: una propuesta de intervención educativa para la atención a la diversidad en niños con retardo en el desarrollo psíquico. Alianza.

Fabra, M. L. (1992). El trabajo cooperativo: revisión y perspectivas, Aula de innovación educativa, 9, 5-12.

Huertas R. Simeón (2005) Estudio titulado: Aprendizaje estratégico en el rendimiento académico de los alumnos de la Facultad de Educación, el proceso metodológico de la enseñanza estratégica en el rendimiento académico. Facultad de Educación de la Universidad Nacional Santiago Antúnez de Mayolo - Huaraz

Johnson, D. W., Johnson, R. T. y Holubec, E. J. (1999). El aprendizaje cooperativo en el aula. Paidós.

Lewin, K. (1973). Dinámica de la personalidad. Morata.

Matamala, R. (2008). Las estrategias metodológicas utilizadas por el profesor de matemática en la enseñanza media y su relación con el desarrollo de habilidades intelectuales de orden superior en sus alumnas y alumnos [Tesis de maestría, Universidad de Chile] Repositorio Académico de la Universidad de Chile. http://eprints.ucm.es/tesis/ edu/ucm-127286.pdf

Skinner, B. F. (1970). Tecnología de la enseñanza. Labor

\section{Contribución de los autores}

MR-A: estrategias metodológicas, análisis de resultados, discusión, revisión final del artículo.

Fuentes de financiamiento

La investigación fue realizada con recursos propios.

\section{Conflictos de interés}

Los autores declaran no tener conflicto de interés.

\section{Correspondencia}

Juan Romelio Mendoza Sanchez

Dirección: Jr. Sucre Nº 604, Cajamarca, Perú. CP. 051

Cel.: 976728581

Email: Juan_mendoza_sanchez@hotmail.com 